\title{
Image-based high strain rate testing of orthopaedic bone cement
}

\author{
Lloyd Fletcher $^{1, *}$, Xavier Régal ${ }^{1}$, Rian Seghir ${ }^{1,2}$, Fabrice Pierron $^{1}$, and Martin Browne ${ }^{1}$ \\ ${ }^{1}$ Engineering and the Environment, University of Southampton, Highfield campus, University Road, Southampton SO17 1BJ, UK \\ ${ }^{2}$ Ecole Centrale de Nantes, 1 rue de la Noë, 44321 Nantes Cedex 3, France
}

\begin{abstract}
Bone cement is widely used for the fixation of orthopaedic implants. It is a multi-component material that consists of a PMMA base with a small proportion of (usually ceramic) radiopacifier to enable the cement to be observed by X-ray. Bone cement is formed through an exothermic reaction in which a powder of pre-polymerised beads of PMMA reacts with MMA monomer. The resulting polymer microstructure consists of PMMA beads in a matrix of newly formed PMMA containing radiopacifier particles. In service, bone cement can experience deformation over a range of strain rates, from the lower end in normal gait to $100 \mathrm{~s}_{\text {of s}}^{-1}$ in the case of falls or impacts. In the current study, it is hypothesised that the response of homogeneous (clear) PMMA to high strain rates will be different to that of bone cement due to the microstructural differences. There have been very few studies on this topic in the past, mostly because of the difficulty involved in adapting the Hopkinson bar protocol to this material, particularly for dynamic tension. The objective of this paper is to present new results on the stiffness and damping of bone cement at strain rates in the range of $100 \mathrm{~s}^{-1}$, and to compare the data with that obtained on clear PMMA. The technique employed here to measure the mechanical properties of both commercial grade PMMA and bone cement is a new image-based DMTA method recently proposed by Seghir and Pierron (Seghir, Pierron, Exp. Mech., 2018). This allows for the measurement of the complex modulus over a range of temperatures and strain rates $\left(100 \mathrm{~s}^{-1} \mathrm{~s}^{-1}\right)$. The method relies on imaging the deformation of the specimen bearing a printed grid using a Shimadzu HPV-X camera at up to 5 million frames per second. This allows for the time-resolved displacements to be measured, leading to fields of strain and acceleration, the latter being used to derive stress information to build up stress-strain curves. The methodology is described in more details in www.photodyn.org.
\end{abstract}

\section{Introduction}

Bone cement is widely used for the fixation of orthopaedic implants. It is a multi-component material that consists of a PMMA base with a small proportion of (usually ceramic) radiopacifier to enable the cement to be observed by X-ray. Bone cement is formed through an exothermic reaction in which a powder of prepolymerised beads of PMMA reacts with MMA monomer. The resulting polymer microstructure consists of PMMA beads in a matrix of newly formed PMMA containing radiopacifier particles. Commercial PMMA and bone cement both consist of pre- polymerized PMMA beads, MMA monomer, and an initiator. However, commercial PMMA is cured (usually in the form of sheets) over a longer timescale (over 10 hours) under high pressure and temperature, while bone cement is injected into the bone canal during an operation at relatively low pressure and temperature, and is cured in less than 15 minutes to fix the prosthesis in place.

In service, bone cement can experience deformation over a range of strain rates depending on the activity of the patient. It is important that the cement can withstand these loading regimes as failure of the cement can eventually lead to loosening of the implant, thus requiring a revision operation where the implant (and the cement) is removed and a new implant is inserted. The two most common mechanisms of cement failure under typical in service loading conditions are initiation at pores (which are almost inevitable in the cement mantle) and initiation at radiopacifier particles. While bone cement is likely to experience low strain rates in service, exceptional events can result in significant strain rates, from falling through to the worst case of a vehicle crash (where strain rates can reach on average $50 \mathrm{~s}^{-1}$ during global deformation of the structure, and up to $500 \mathrm{~s}^{-1}$ in component based models).

In the current study, it is hypothesised that the response of homogeneous (clear) PMMA to high strain rates will be different to that of bone cement due to the microstructural differences highlighted above. There have been very few studies on this topic in the past, mostly because of the difficulty involved in adapting the Hopkinson bar protocol to this material, particularly for dynamic tension. However, a recent paper [1] has demonstrated the effect of strain rate on bone cement

*Corresponding author: 1.c.fletcher@soton.ac.uk 
and found that the fracture behaviour was similar in quasi-static between a commercial grade PMMA and PMMA-based bone-cement but quite different at high strain rates. The objective of this paper is to present new results on the stiffness of bone cement at strain rates in the range of $100 \mathrm{~s}$ of $\mathrm{s}^{-1}$, and to compare the data with that obtained on clear PMMA.

\section{Experimental set-up and data processing}

\subsection{Experimental set-up}

The experimental set-up is represented in figure 1. It consists of the following:

- A NextGenLab 750 high-power ultrasonic transducer system from SynapTec (France) which allows for the sample to by cyclically loaded at $20 \mathrm{kHz}$ up to a peak-to-peak displacement amplitude of 120 $\mu \mathrm{m}$, depending on the sample design and damping.

- A Cedip Silver 480M infrared (IR) camera that measures the heat flux radiated by the deforming sample and reconstructs space resolved surface temperature fields. The IR camera has a resolution of $320 \times 256$ pixels, and is used here at a frame rate of 50 Hz. This will not al- low for the temperature to be followed during the few ultrasonic runs used to image the deformation, but will provide the gradual heating up during the successive ultrasonic runs.

- A HyperVision HPV-X ultra-high speed camera from the Shimadzu Corporation, which can record 128 images at frame rates up to $10 \mathrm{Mfps}$ with a spatial resolution of $400 \times 250$ pixels regardless of the frame rate. Here, images will be captured at $500 \mathrm{kfps}$ providing 25 images per $20 \mathrm{kHz}$ cycle for a total of 5 imaged cycles.

- A rectangular bone cement specimen of dimensions $46 \mathrm{~mm}$ in length, $15 \mathrm{~mm}$ in width and 3 $\mathrm{mm}$ in thickness. The specimens were prepared using a PTFE mould. One face of the specimen was equipped with a grid of pitch $0.9 \mathrm{~mm}$ obtained by dry transfer, as detailed in [2]. The other side of the specimen was covered by black matt paint to obtain a large emissivity coefficient.

The specimen was bonded onto the tip of the sonotrode using cyanoacrylate glue. It was then submitted to several ultrasonic runs. Each run lasted 0.8 $\mathrm{s}$, over which, approximately after $0.4 \mathrm{~s}$, five loading cycles were recorded with the HPV-X camera. The specimen was then left to cool down until the images have been downloaded to the computer and another run could start. Runs have been con- ducted until the specimens failed or collapsed.

The specimen length was determined so that its first longitudinal resonance frequency is $20 \mathrm{kHz}$. Therefore, when loaded, it vibrates such that the strain is maximal at its centre and zero at both ends. Because of dissipation of mechanical energy into heat during each loading cycle, heat build ups in the specimen with the maximum at its centre. Therefore, each section of the sample sees a different strain, strain rate and temperature as we sweep through the runs until the specimen fails or collapses.

\subsection{Data processing}

From the grey level grid images, spatial phase shifting was used to produce phase maps which then provided displacements for each pixel of the image. This is known as the 'Grid Method' and readers are referred to a recent review paper on this topic [3]. The reason for using this technique instead of Digital Image Correlation (DIC) is that it provides a better compromise between resolution in dis- placement and spatial resolution, as demonstrated in [4]. Once displacement maps were available, spatial differentiation was used to obtain the three components of strain, and double temporal differentiation was applied to derive acceleration. This required some amount of temporal and spatial smoothing and the reader is referred to [2] for more details.

The key idea in this test is to use acceleration fields to reconstruct average longitudinal stress at each transverse section where a data point is available. The complete derivation is available in [2]. Since the stress state is uniaxial, one can plot average stress versus average strain and identify stiffness. More precisely here, both average re- constructed longitudinal stress and average measured longitudinal strain were projected onto sine functions in time allowing for the storage modulus and $\tan (\delta)$ to be identified [2].

\section{Results}

This section presents some comparative results between bone cement and clear PMMA, which data are taken from [2]. Figure 2a shows stress-strain curves obtained at three different sections at the end of the series of runs, when the temperature at the centre of the specimen was about $80^{\circ} \mathrm{C}$. Because the specimen heats up in the middle, one can see that the response there is less stiff and has a larger amount of mechanical energy dissipated (damping) than the other two sections. The one closest to the free edge exhibits the largest modulus and the lowest $\tan \delta$ ) while the section in between has intermediate behaviour. The difference of behaviour between bone cement and clear PMMA is already very apparent on the raw thermal images in figure $2 \mathrm{~b}$. While clear PMMA exhibits a smooth temperature distribution, bone cement shows a much more concentrated hot spot as well as some smaller local hot spots. This is consistent with the fact that contrary to clear PMMA which has a homogeneous structure at the mesoscale, bone cement is polymer - polymer composite with porosities and radioopacifier, hence heterogeneous at the mesoscale.

Figure 3a graphically represents all storage modulus values obtained from the different sections and runs. Each point on the plot represents one section at a particular run, and the colour map has been generated by interpolation between these points. As expected, the modulus increases with temperature. Over the small strain rate range considered here, the variation of modulus with strain rate is very small, though there is a 


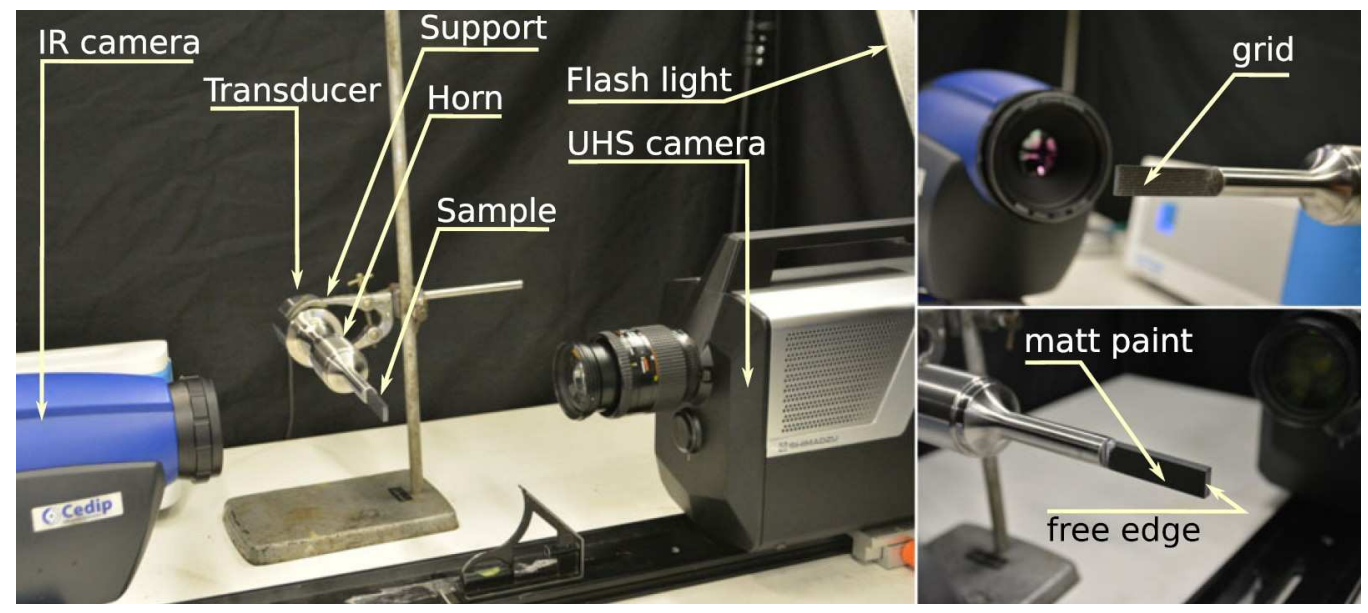

Fig. 1. Experimental set-up for the image-based DMTA.

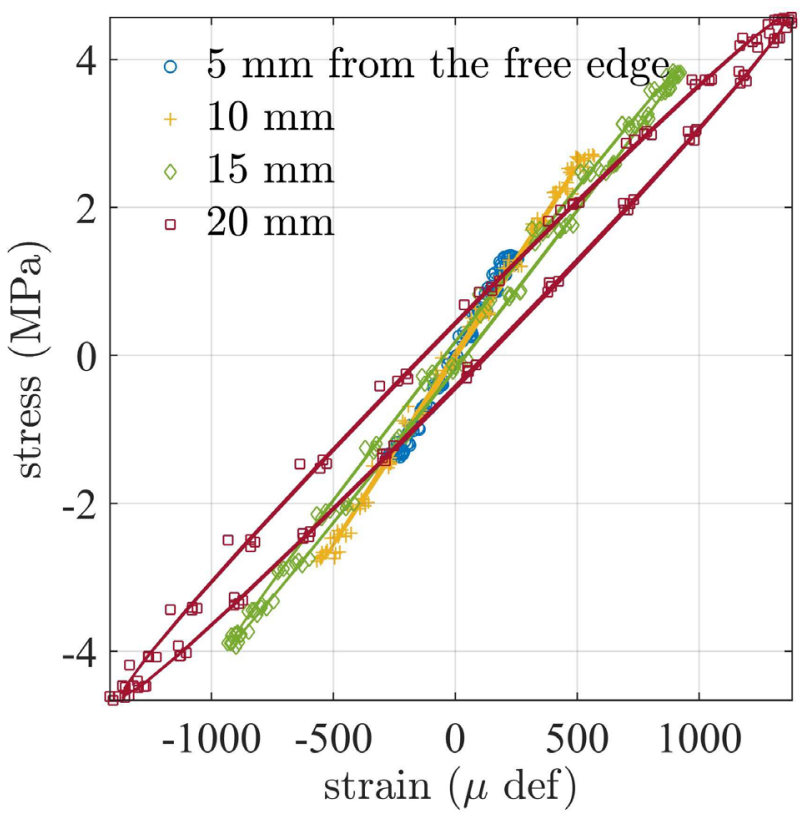

(a) Stress-strain curves for three sections of the specimen, the markers represent data points and the line is obtained from the fitted stress and strain in time.
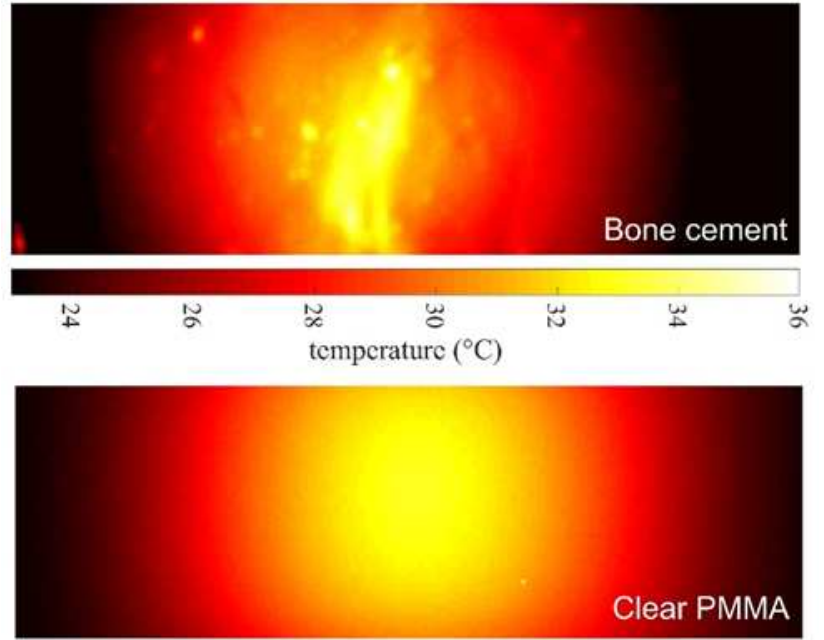

(b) Comparison of temperature distributions during the tests between bone cement and clear PMMA.

Fig. 2. Stress-strain curves for the bone cement and temperature field comparison between bone cement and clear PMMA.

strange trend of slight modulus decrease with strain rate. This could be caused by the fact the points at low temperatures and higher strain rates correspond to the central sections during the first couple of runs, and the strains are much larger there than towards the edges (points at low temperature and lower strain rates). It is well-known in quasi-static that PMMA exhibits nonlinear behaviour in its elastic range, with stiffness decreasing with strain levels. This may also be the case here though more results would be needed. Comparing the map to that obtained on clear PMMA in figure $3 \mathrm{~b}$, one can see that the storage modulus varies much less with temperature than for clear PMMA. From room temperature up to $70^{\circ} \mathrm{C}$, clear PMMA is slightly stiffer than bone cement but this trend reverts at higher temperature. This may indicate a higher glass transition temperature $(\mathrm{Tg})$ for bone cement, though this value could not be reached because of the failure of the bone cement specimens at around $90^{\circ} \mathrm{C}$. Figure 4 reports the damping parameter $\tan (\delta)$ in the same format. The difference here is striking, with much lower damping for bone cement and a very small variation with temperature.

Finally, figure 5 illustrates the failure modes of both materials. While clear PMMA forms cavitation bubbles as the temperature reaches its $\mathrm{Tg}$, bone cement exhibits a brittle-like failure at the centre well away from its $\mathrm{Tg}$ as no steep stiffness reduction was recorded (figure $3 \mathrm{a}$ ), nor strong increases in $\tan (\delta)$. The fracture surface is rough and one can clearly see the small PMMA beads on the picture. This brittle-like behaviour is consistent with the 


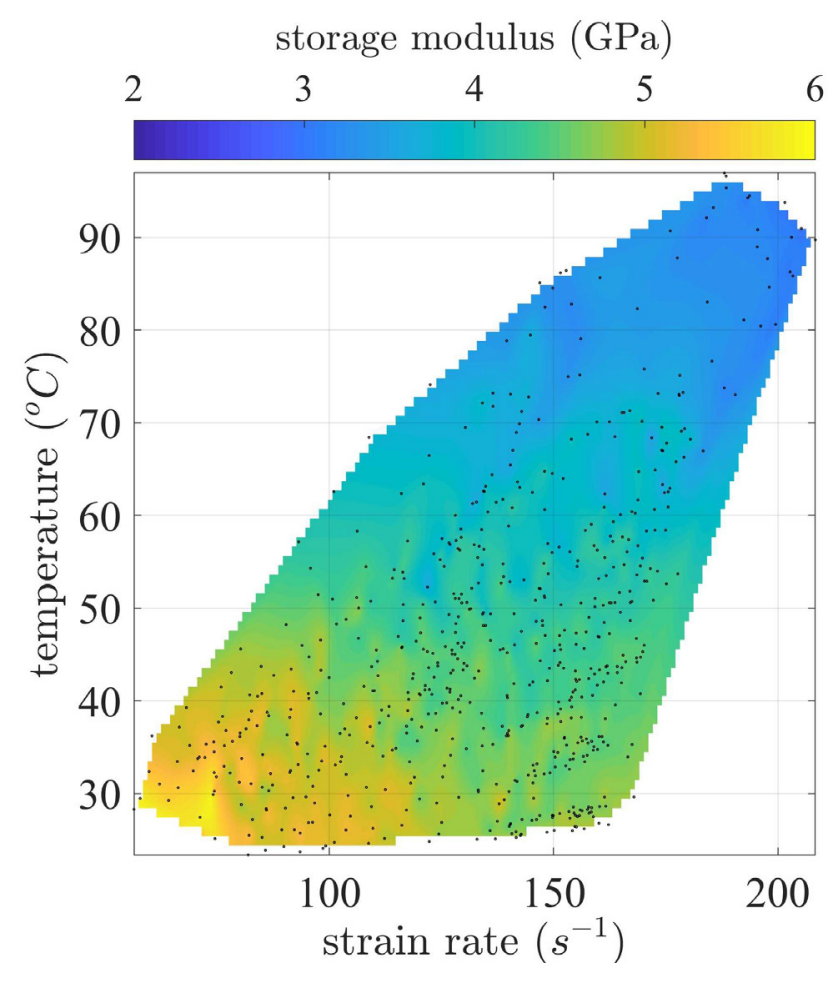

(a) Bone cement

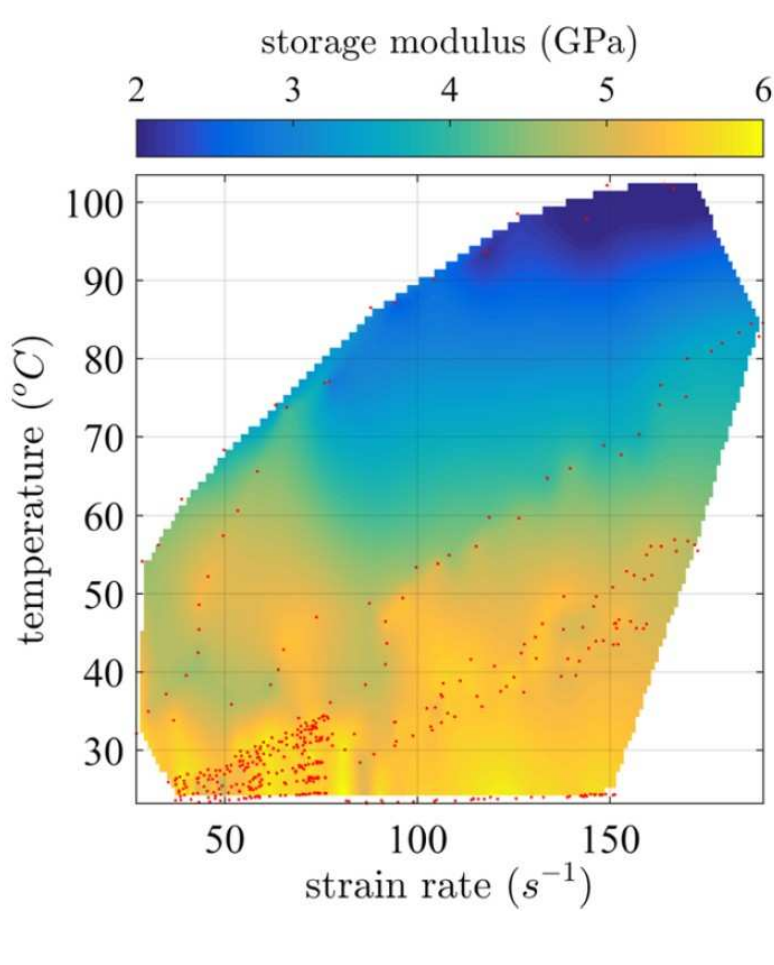

(b) Clear PMMA

Fig. 3. Identification of the storage modulus as a function of strain-rate and temperature, for bone cement and clear PMMA.

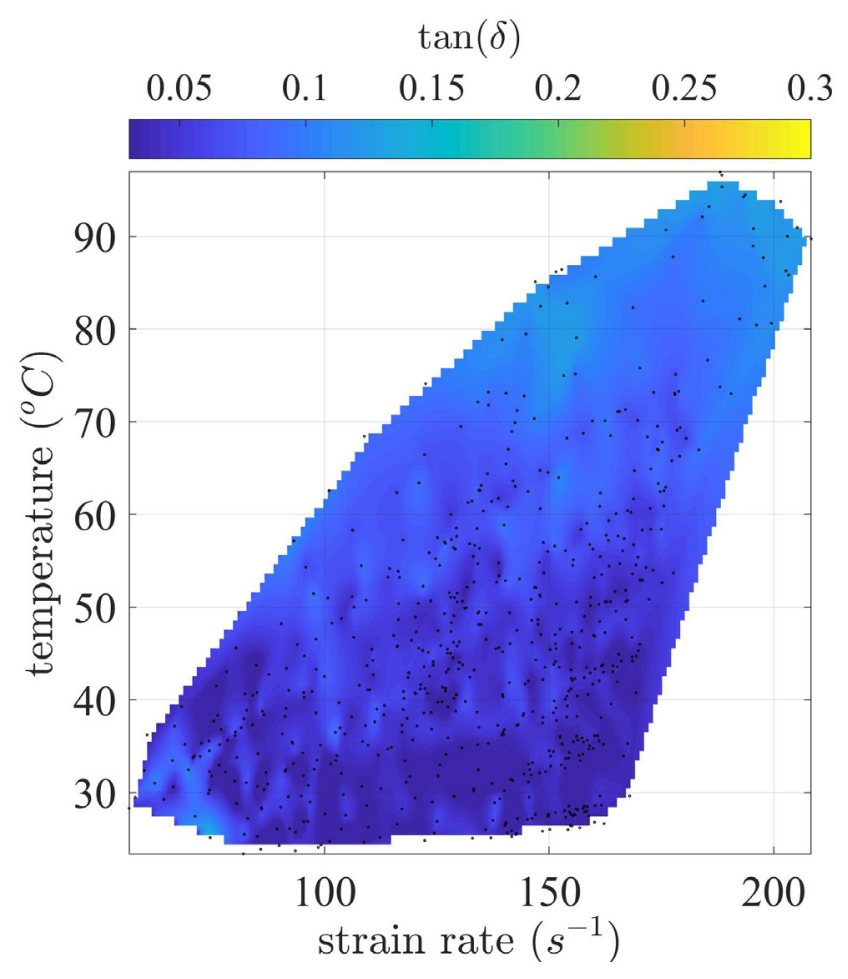

(a) Bone cement

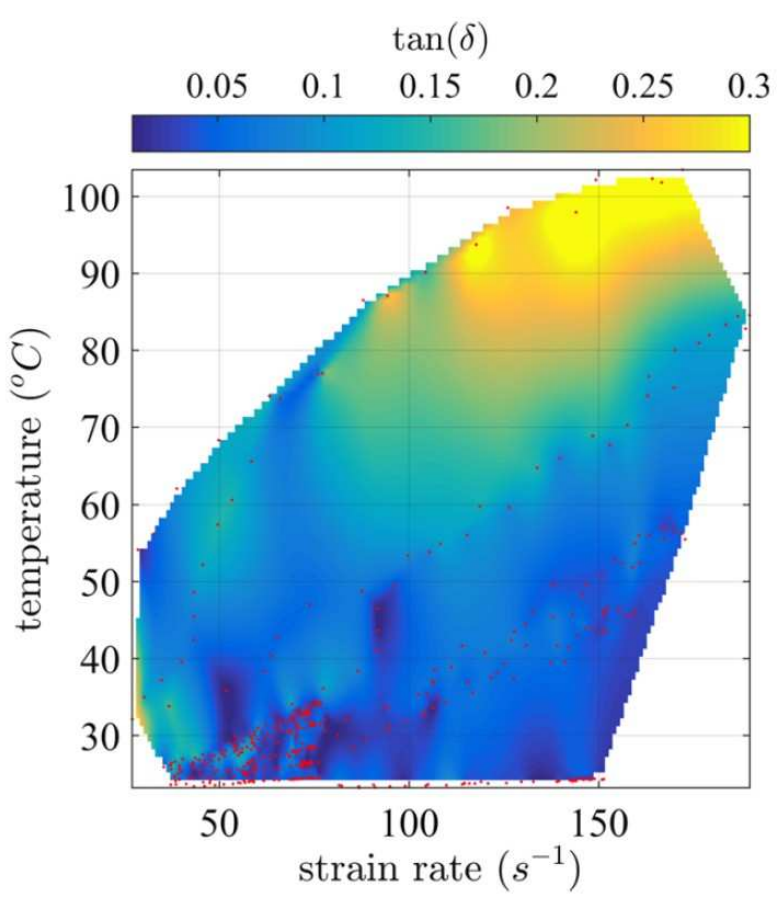

(b) Clear PMMA

Fig. 4. Identification of $\tan (\delta)$ as a function of strain-rate and temperature, for bone cement and clear PMMA. 


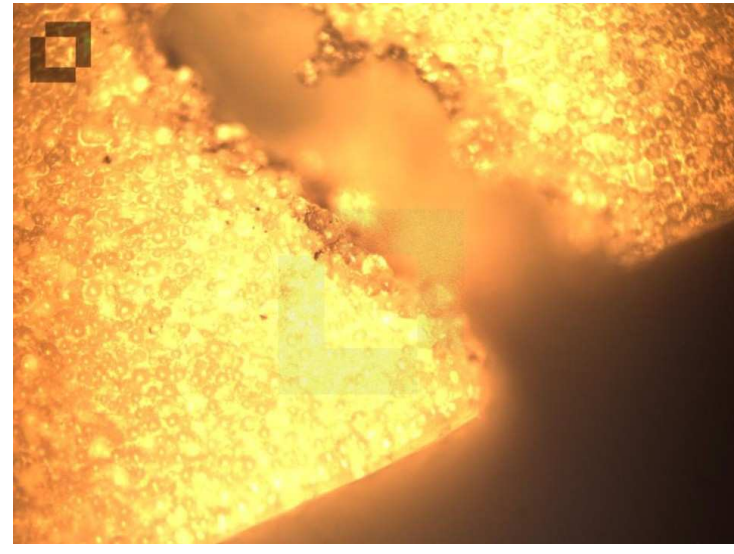

(a) Bone cement

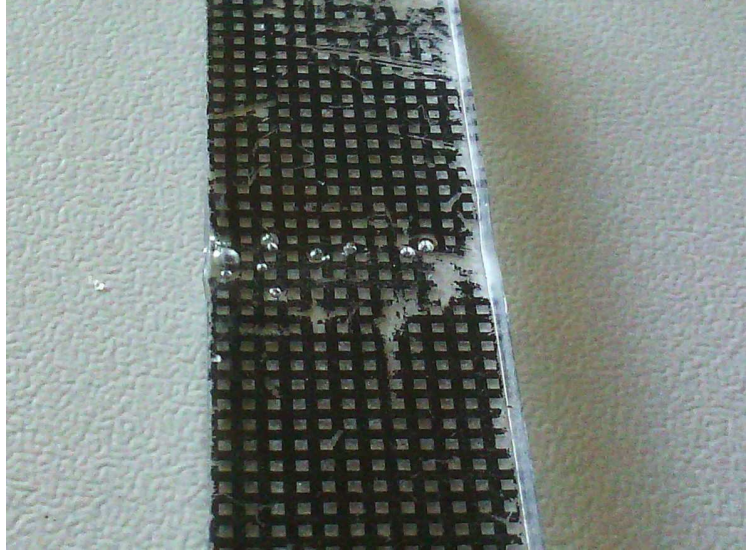

(b) Clear PMMA

Fig.5. Comparative failure modes between bone cement and clear PMMA.

findings in [1] at higher strain rates, as well as the present damping measurements (figure $4 a$ ).

\section{Conclusions and future work}

This study confirms what was observed in [1], that bone cement exhibits a more brittle behaviour than clear PMMA at high strain rates. The thermal images recorded during the present study also showed a more heterogeneous behaviour consistent with the rough fracture surface conditioned by the material heterogeneity. This is a preliminary study and more work is needed to investigate the behaviour of bone cement at high strain rates.

First, initial X-ray CT scanning of the specimens would enable the density of porosities to be measured as well as the clustering of radio-opacifier particles to be evaluated, as both phenomena are believed to play a major role in the behaviour of the bone cement. More specimens need to be tested, ideally with varying amounts of radio- opacifier and proportions of PMMA beads to PMMA matrix. Finally, inertial impact tests (IBII, see [5]) would allow for the behaviour at even higher strain rates to be evaluated, and fracture strength to be measured accurately and compared to that of clear PMMA.

Funding from EPSRC, grant EP/L026910/1, is gratefully acknowledged. The authors also thank Mr Adam Dakin, undergraduate student at the University of Southampton, for preparing the test specimens.

\section{References}

1. R. Bedsole, H.V. Tippur, Journal of Engineering Materials and Technology 135, 031001 (2013)

2. R. Seghir, F. Pierron, Experimental Mechanics 58, 183 (2018)

3. M. Grédiac, F. Sur, B. Blaysat, Strain 52, 205 (2016)

4. M. Grédiac, B. Blaysat, F. Sur, Experimental Mechanics 57, 871 (2017)
5. L. Fletcher, J. Van Blitterswyk, F. Pierron, Composites Part A: Applied Science and Manufacturing Submit-ted. (2018) 
